\title{
PERFIL SOCIOECONÔMICO, DEMOGRÁFICO E INDICATIVO DE DEPRESSÃO EM MULHERES SUBMETIDAS À MASTECTOMIA NO PÓS-OPERATÓRIO TARDIO
}

\section{SOCIOECONOMIC, DEMOGRAPHIC PROFILE AND INDICATIVE OF DEPRESSION IN WOMEN UNDERGOING MASTECTOMY IN THE LATE POSTOPERATIVE PERIOD \\ PERFIL SOCIECONÓMICO, DEMOGRÁFICO Y INDICATIVO DE LA DEPRESIÓN EM LAS MUJERES SOMETIDAS A MASTECTOMÍA EM EL POSTOPERATORIO TARDÍO}

Jaciara Tiago Antunes Alvarenga ${ }^{1}$, Nayara Paula Fernandes Martins Molina ${ }^{2}$, Grazielle Riceto Dias Silva $^{3}$, Lilane Maria Alves Silva ${ }^{4}$, Leiner Resende Rodrigues ${ }^{5}$

\begin{abstract}
RESUMO
Objetivo: descrever as características socioeconômicas e demográficas e avaliar o indicativo de depressão em 30 mulheres mastectomizadas no pós-operatório tardio. Método: pesquisa quantitativa e transversal, realizada no Hospital de Clínicas da Universidade Federal do Triângulo Mineiro, Uberaba - MG. Utilizou-se o Inventário de Depressão de Beck; e para a caracterização geral, instrumento elaborado pelas autoras. Resultados: a média de idade foi 63,4 anos; maioria viúvas; escolaridade entre 1 a 4 anos; dois filhos; composição familiar de 2 e 3 pessoas; residentes em casa própria; religião católica; maior parte aposentada ou pensionista; e renda de 01 salário mínimo. Quanto às variáveis de saúde: sem antecedente de doença mental; não realização de acompanhamento psicológico; sem dificuldade para executar tarefas domésticas; sem reconstrução da mama. A média no Inventário de Depressão de Beck foi 15,3 $\pm 11,04$ pontos. Conclusão: destaca-se a importância do acompanhamento dessas mulheres por meio de um cuidado holístico, individualizado e humanizado.

Descritores: Saúde mental; Depressão; Enfermagem; Mastectomia; Período Pós-Operatório.
\end{abstract}

\begin{abstract}
Objective: to describe the socioeconomic and demographic characteristics and evaluate the indicative of depression in 30 women undergoing mastectomy in late postoperative period. Method: quantitative and cross-sectional survey conducted at the Clinical Hospital of the Federal University of Triângulo Mineiro, Uberaba - MG. The Beck Depression Inventory was used; and for the general characterization, an instrument was developed by the authors.

\footnotetext{
${ }^{1}$ Enfermeira pela UFTM. E-mail: jaciera_@ hotmail.com

${ }^{2}$ Enfermeira. Doutora em Atenção à Saúde. UFTM. E-mail: nayara.pfmartins@gmail.com

${ }^{3}$ Enfermeira. Mestre em Atenção à Saúde pela UFTM. E-mail: graziellerd@yahoo.com.br

${ }^{4}$ Enfermeira. Mestre em Atenção à Saúde pela UFTM. E-mail: graziellerd@ yahoo.com.br

${ }^{5}$ Enfermeira. Doutora em Enfermagem Psiquiátrica. Professora Associada do Departamento de Enfermagem em Educação e Saúde Comunitária do Curso de Graduação em Enfermagem. Universidade Federal do Triângulo Mineiro (UFTM). E-mail: leiner.r.rodrigues@gmail.com
} 
Results: the mean age was 63.4 years; most widows; with schooling between 1 to 4 years; two sons; family composition of 2 and 3 persons; residents in their own home; Catholic religion; most part retired or pensioner; 01 minimum wage income. As for the health variables: no history of mental illness; no psychological treatment; with no difficulty to perform household chores; no breast reconstruction. The average of Beck Depression Inventory was $15.3 \pm 11.04$ points. Conclusion: the study contributed to elucidate the sociodemographic and health characteristics of women undergoing mastectomy in late postoperative period.

Descriptors: Mental health; Depression; Nursing; Mastectomy; Postoperative Period.

\section{RESUMEN}

Objetivo: describir las características socioeconómicas y demográficas, y evaluar lo indicativo de la depresión en 30 mujeres mastectomizadas en el postoperatorio tardío. Método: estudio cuantitativo y transversal realizado en el Hospital Clínico de la Universidad Federal de Triángulo Mineiro, Uberaba - MG. Se utilizó el Inventario de Depresión de Beck y para la caracterización general, un instrumento desarrollado por los autores. Resultados: la edad media fue de 63,4 años; la mayoría de las viudas; la escolarización entre 1 a 4 años; dos hijos; la composición familiar de 2 y 3 personas; residentes en su propia casa; la religión católica; su mayor parte se retiró o pensionado; y 01 ingreso de salario mínimo. En cuanto a las variables de salud: sin antecedentes de enfermedad mental; sin tratamiento psicológico; sin dificultad para realizar las tareas del hogar; sin reconstrucción de mama. La puntuación media en Inventario de Depresión de Beck fue de 15,3 \pm 11,04 puntos. Conclusión: el estudio contribuyó a dilucidar las características sociodemográficas y de salud de las mujeres sometidas a mastectomía en el postoperatorio tardio.

Descriptores: Salud mental; Depresión; Enfermería; Mastectomía; Postoperatorio.

\section{INTRODUÇÃO}

A mastectomia é um procedimento cirúrgico utilizado para tratamento do câncer de mama, que é o tipo de neoplasia maligna que mais acomete as mulheres, com exceção do câncer de pele não melanoma. Consiste na retirada da mama ou glândula mamária, da pele e complexo aréolo-papilar, podendo estar associada à linfadenectomia axilar e remoção dos músculos peitorais. ${ }^{1}$

Como consequência da extirpação tecidual, mulheres na fase operatória tardia, assim como no período operatório imediato, podem apresentar dor, limitação dos movimentos e linfedema. ${ }^{2}$ Entretanto, na fase tardia, as dificuldades relacionamse mais com o autocuidado, produtividade e lazer (isto inclui apresentar baixa ou média autoestima $)^{3}$ e prejuízo da rotina (manter domicílio, conduzir veículos, escrever e digitar, utilizar transporte coletivo, lavar roupas, fazer compras, preparar alimentos, manter relação sexual, realizar passeios com a família, participar de atividades sociais, praticar atividade física e realizar atividades artesanais). ${ }^{4}$ 
Além disso, muitas vezes há necessidade de mudança de função ou do tipo de atividade produtiva e diminuição de renda. $^{4}$

Por ser um tratamento mutilador, a mastectomia é responsável por causar na mulher uma percepção negativa em relação à sua imagem corporal, o que ocasiona problemas na função sexual e comprometimento da sua representação simbólica - feminilidade e maternidade. ${ }^{5}$ Estas mudanças e alterações na autoestima e no sentimento de feminilidade podem favorecer o aparecimento de sintomas depressivos. ${ }^{6}$ A sintomatologia depressiva acomete até $25 \%$ das mulheres que se submetem à mastectomia. ${ }^{7}$

A Organização Mundial de Saúde ${ }^{8}$ considera a depressão um grave problema de Saúde Pública e estima que 151 milhões de pessoas sejam vítimas da mesma. Assim como todas as fases pelas quais a mulher passa desde o diagnóstico do câncer de mama, o período pós-operatório também gera forte impacto emocional e traz a necessidade de enfrentamento e adaptação à nova situação e assimilação das perdas, visto que com a cirurgia a mulher corre maior risco de desenvolver sintomas depressivos. ${ }^{9}$ Mediante esse cenário de possível fragilidade emocional, e também à reduzida atenção despendida à mulher na fase tardia da mastectomia, em comparação com o pós-operatório imediato, justifica-se a realização do presente estudo.

Diante do exposto, os objetivos desse estudo foram: descrever as características socioeconômicas e demográficas das mulheres mastectomizadas no pós-operatório tardio e avaliar a presença de indicativo de depressão nestas mulheres.

\section{MÉTODO}

Trata-se de uma pesquisa quantitativa, de caráter descritivo e transversal, realizada com mulheres submetidas à cirurgia de Mastectomia, no período de $1^{\circ}$ de Janeiro de 2000 a 31 de Dezembro de 2012, período estipulado levando-se em conta a necessidade de a população se enquadrar no período de pósoperatório tardio (a partir de seis meses) no Hospital de Clínicas da Universidade Federal do Triângulo Mineiro, em Uberaba - MG. A amostra de 67 mulheres foi calculada com nível de confiança de 95\%, em uma população de 80 mulheres submetidas à mastectomia que se encontravam no período do pós-operatório tardio. Estes números foram colhidos dos 
prontuários do arquivo do Hospital de Clínicas referido, tendo a amostra sido selecionada de maneira aleatória.

Utilizou-se um instrumento elaborado pelas próprias pesquisadoras acerca das questões socioeconômicas e demográficas e de saúde em geral (antecedente de doença mental, realização de acompanhamento psicológico e de reconstrução da mama).

Para avaliar o indicativo de depressão, utilizou-se o Inventário de Depressão de $\operatorname{Beck}^{10}$ que é um instrumento que visa rastrear a presença de sintomas depressivos em indivíduos de um estudo. Ele possui 21 questões que avaliam e classificam as atitudes e sintomas depressivos que refletem o estado presente do sujeito. São avaliados sentimentos de tristeza, ideação suicida, pessimismo, autodepreciação, autoacusação, fracasso, culpa, perda de autossatisfação, irritabilidade, punição, crises de choro, indecisão, isolamento social, inibição para o trabalho e distorção da imagem corporal. O escore total varia de 0 a 63 pontos.

Os dados foram coletados por meio de entrevista na residência das mulheres selecionadas, no período de Maio a Outubro de 2015, por pesquisadores (alunos de graduação e pós-graduação stricto sensu da área da saúde) previamente treinados. $\mathrm{O}$ agendamento das entrevistas foi realizado por meio de contato telefônico, no intuito de marcar o melhor dia e horário para a realização. Posteriormente, as entrevistas foram avaliadas pelo supervisor (docente responsável) de campo quanto ao seu preenchimento e consistência dos itens para a garantia do controle de qualidade.

Foram consideradas perdas: 11 falecidas, 21 que mudaram da cidade de Uberaba (MG), 13 que não foram encontradas após três tentativas e 5 que se recusaram a participar da pesquisa, chegando-se a uma amostra final composta por 30 mulheres.

A análise estatística foi realizada por meio da distribuição de frequências absolutas e percentuais para variáveis categóricas, e média e desvio padrão para as variáveis numéricas, utilizando-se o aplicativo SPSS "Statistical Package for the Social Sciences" 17.0.

O projeto foi aprovado pelo Comitê de Ética em Pesquisa da Universidade Federal do Triângulo Mineiro sob o número 698.178, e a entrevista só foi iniciada após a anuência e assinatura do Termo de Consentimento livre e esclarecido. 


\section{Resultados}

Das mulheres entrevistadas a média de idade foi $63,4 \pm 8,98$, com mínimo de 50 e máximo de 85 anos. A média de tempo de realização de cirurgia de mastectomia foi de 8,3 anos. A maior porcentagem relatou ser viúvas (40\%), ter escolaridade entre 1 a 4 anos (43,3\%), ter dois filhos (40\%), composição do grupo familiar de 2 $(30,0 \%)$ e $3(30,0 \%)$ pessoas e ter religião católica $(63,3 \%)$, como demonstra a Tabela 1.

Tabela 1 - Distribuição das frequências das características sociodemográficas de mulheres no pós-operatório tardio de mastectomia, Uberaba - MG, 2015.

\begin{tabular}{|c|c|c|}
\hline Variáveis & $\mathbf{N}$ & $\%$ \\
\hline \multicolumn{3}{|l|}{ Estado civil } \\
\hline Solteira & 02 & 6,7 \\
\hline Casada & 10 & 33,3 \\
\hline Viúva & 12 & 40,0 \\
\hline Divorciada & 05 & 16,7 \\
\hline União estável & 01 & 3,3 \\
\hline \multicolumn{3}{|l|}{ Escolaridade (anos de estudo) } \\
\hline Sem escolaridade & 01 & 3,3 \\
\hline 1 a 4 & 13 & 43,3 \\
\hline 5 a 9 & 08 & 26,7 \\
\hline 10 a 12 & 06 & 20,0 \\
\hline 13 anos ou mais & 02 & 6,7 \\
\hline \multicolumn{3}{|l|}{ Número de filhos } \\
\hline Nenhum & 1 & 3,3 \\
\hline 01 & 02 & 6,7 \\
\hline 02 & 12 & 40,0 \\
\hline 03 & 06 & 20,0 \\
\hline 04 ou mais & 09 & 30,0 \\
\hline \multicolumn{3}{|l|}{ Composição do grupo familiar } \\
\hline 01 & 05 & 16,7 \\
\hline 02 & 09 & 30,0 \\
\hline 03 & 09 & 30,0 \\
\hline 04 & 04 & 13,3 \\
\hline 05 & 03 & 10,0 \\
\hline \multicolumn{3}{|l|}{ Religião } \\
\hline Católico & 19 & 63,3 \\
\hline Espírita & 04 & 13,3 \\
\hline Testemunha de Jeová & 01 & 3,3 \\
\hline Evangélico & 06 & 20,0 \\
\hline
\end{tabular}

A maioria das mulheres não exerce atividade remunerada, apenas o faziam antes da cirurgia de mastectomia $(56,7 \%)$, e entre as que exercem atividade remunerada $(16,7 \%)$, a maior parte $(60 \%)$ executa carga horária semanal de 20 a 40 horas. Em relação à participação na vida econômica do grupo familiar a maior 
porcentagem dessas mulheres não trabalha, é aposentada/pensionista e contribui com a família $(46,7 \%)$. A maioria das mulheres mastectomizadas na fase tardia possui renda bruta de 01 salário mínimo $(73,3 \%)$ e o tipo de habitação predominantemente é casa própria $(83,3 \%)$.

A maior porcentagem não apresentava antecedentes de doença mental (70,0\%), não realizou acompanhamento psicológico antes ou após o câncer de mama (43,3\%), Tabela 2. Entretanto entre aquelas que disseram que realizaram acompanhamento psicológico, o maior percentual $(36,7 \%)$ referiu ter feito após a cirurgia, seguida daquelas que fizeram terapia após descobrir o câncer $(16,7 \%)$ e somente $3,3 \%$ referiu já realizar acompanhamento terapêutico antes de descobrir o câncer de mama.

Destaca-se que as mulheres mantiveram as tarefas domésticas da mesma forma que antes da cirurgia de mastectomia (90,0\%), não apresentaram nenhum grau de dificuldade para realização das tarefas domésticas $(40,0 \%)$ e não realizaram reconstrução da mama $(73,3 \%)$, de acordo com os dados da tabela 2.

Tabela 2 - Distribuição das frequências dos aspectos relacionados à saúde mental e realização de tarefas domésticas de mulheres no pós-operatório tardio de mastectomia, Uberaba - MG, 2015.

\begin{tabular}{lcc}
\hline \multicolumn{1}{c}{ Variáveis } & $\mathbf{N}$ & $\mathbf{\%}$ \\
\hline Antecedente de doença mental & 21 & 70 \\
Não & 09 & 30 \\
Sim & & \\
Acompanhamento psicológico & 13 & 43,3 \\
Não & 17 & 56,7 \\
Sim & & \\
Manutenção das tarefas domésticas da mesma forma que & & \\
antes da cirurgia de mastectomia & 03 & 10,0 \\
Não & 27 & 90,0 \\
Sim & & \\
Grau de dificuldade para realização das tarefas domésticas & 12 & 40,0 \\
Não & 15 & 50,0 \\
Sim & 03 & 10,0 \\
Ignorado/não respondido & & \\
Reconstrução da mama & 22 & 73,3 \\
Não & 08 & 26,7 \\
Sim & & \\
\hline
\end{tabular}


A média da pontuação no Inventário de Depressão de Beck foi $15,3 \pm 11,04$ e a mediana 13 pontos.

Discussão

A maioria das mulheres participantes deste estudo eram viúvas (40\%). Em estudo similar, o estado conjugal predominante foi de mulheres casadas $(70 \%)$, com faixa etária inferior (41 a 50 anos $)^{11}$, idade esta em que as mulheres normalmente vivem com o companheiro. No presente estudo prevaleceram mulheres idosas, com média de idade de $63,4 \pm 8,98$ anos.

Muitas vezes o relacionamento conjugal pode sofrer distanciamento nesse período e acabar por afetar o emocional destas mulheres, deixando-as mais fragilizadas ainda. ${ }^{12}$ Por outro lado, o esposo muitas vezes pode adquirir a função de cuidador principal, se tornando mais atencioso e prestativo após a cirurgia de mastectomia, inclusive tendo papel fundamental na fase de luto pela retirada da mama, ajudando-a no enfrentamento deste problema. ${ }^{13} \mathrm{Em}$ ambos os casos, a enfermagem deve orientar tanto a mulher como seu companheiro a compartilhar seus sentimentos frente à mastectomia, trabalhando as alterações provocadas pela doença na vida conjugal. ${ }^{13}$
O nível de escolaridade predominante foi de um a quatro anos de estudo (43,3\%), o que remete a dados expressos na literatura, em que prevalece o ensino fundamental incompleto. ${ }^{4,14}$ A escolaridade pode interferir no cuidado com a saúde, uma vez que quanto mais tempo de estudo, maior a instrução e consequentemente maior compreensão sobre o processo saúde/doença. ${ }^{12}$ Com base nisso, a equipe multiprofissional deve promover a educação em saúde, incentivando o autocuidado.

O presente estudo evidenciou como dois o número de filhos da maior porcentagem das mulheres mastectomizadas (40\%), sendo tal dado semelhante à pesquisa conduzida no Mato Grosso. ${ }^{11}$ É destaque que o apoio ofertado pelos familiares é importante tanto no momento da descoberta da doença, como em todas as outras fases, que envolvem desde a cirurgia até o pós-operatório de mastectomia, fazendo com que a mulher receba suporte desde suas necessidades básicas até o desempenho de seus papéis. ${ }^{15}$

A maior porcentagem das pesquisadas apresentou composição do grupo familiar de duas $(30 \%)$ e três $(30 \%)$ pessoas. Muitas vezes quem realiza o cuidado à mulher mastectomizada é a 
família, tornando o mesmo um desafio para as partes, pois envolve 0 relacionamento interpessoal, demonstrando que o cuidar sofre influências de significado e consequentemente de condutas. Muitas vezes o acesso desses familiares a questões subjetivas da mulher mastectomizada é muito mais efetivo que o do profissional de saúde ${ }^{16}$, tornando o familiar um aliado da equipe de saúde no processo de cuidar.

Em relação à religiosidade, todas as mulheres relataram haver alguma crença religiosa na qual buscam apoio. No presente estudo a religião católica foi predominante $(63,3 \%)$ estando em conformidade com dados apresentados por outros autores. ${ }^{12}$ As crenças religiosas são uma das formas de as mulheres buscarem forças frente ao caráter ameaçador do câncer de mama, para através delas, encarar o sofrimento e estigma social, lidando com um cotidiano onde a doença imputa marcas psicossociais. ${ }^{17}$

Referente ao trabalho, a maioria das mulheres no pós-operatório tardio de mastectomia não exercia mais atividade remunerada, apenas antes da cirurgia de $(56,7 \%)$, fato esperado devido à faixa etária em que elas se encontram (média de $63,4 \pm 9,0$ anos), condizente com a aposentadoria. Tal dado discerne de um estudo realizado no nordeste brasileiro, em que a maioria das mulheres manteve o mesmo trabalho após a cirurgia de mastectomia $(61,6 \%)$, situação esta que pode ser atribuída à faixa etária na qual se encontram (50 a 59 anos - 47,6\%). Entretanto, chama a atenção que $62,5 \%$ necessitaram de mudança de função para continuar trabalhando. ${ }^{4}$

Com relação à participação na vida econômica do grupo familiar, $46,7 \%$ das mulheres mastectomizadas no pósoperatório tardio não trabalhavam, eram aposentadas ou pensionistas e contribuíam com a família, antagonizando estudo prévio, o qual relata que a maior porcentagem (50\%) das mulheres mastectomizadas trabalhavam e eram funcionárias públicas. Tal fato pode ser sugerido possivelmente pela faixa etária em que a maior porcentagem delas se encontrava, de 41 a 50 anos, sendo mais jovens e ainda economicamente ativas. ${ }^{11}$ Salienta-se que a presença de limitações físicas oriundas da mastectomia pode resultar no abandono do mercado de trabalho formal favorecendo a inserção no mercado informal, com o intuito de complementar a renda familiar, defasada pela perda do emprego. ${ }^{12}$ Nessa nova 
realidade, a enfermagem deve se mostrar qualificada em atender as necessidades de saúde dessas mulheres, buscando orientar na readaptação das atividades rotineiras.

A maioria das mulheres mastectomizadas no pós-operatório tardio referiu possuir renda bruta predominante de um salário mínimo $(73,3 \%)$ e casa própria $(83,3 \%)$. Tais dados concordam com outros estudos envolvendo a temática, como o conduzido em Salvador - Bahia, cuja população foi composta por 98 mulheres mastectomizadas e que reportou que $67,3 \%$ das mulheres referiu renda inferior ou igual a um salário mínimo. ${ }^{2}$ De maneira similar, estudo com 62 pacientes em um hospital do Rio de Janeiro evidenciou que $30,6 \%$ das mulheres recebiam até um salário mínimo. ${ }^{14}$

A maioria das mulheres não apresentou antecedente de doença mental (70\%) e a maior porcentagem não realizou acompanhamento com psicólogo em nenhuma das fases do tratamento cirúrgico $(43,3 \%)$. Outra pesquisa, todavia, trouxe como maior porcentagem mulheres mastectomizadas que receberam acompanhamento psicológico no pósoperatório $(45 \%)$, seguido por mulheres que receberam acompanhamento psicológico no pré e pós-operatório $(30 \%)$ e $25 \%$ que não o receberam em nenhum momento. ${ }^{11}$ Existe uma grande demanda para o profissional psicólogo para com essas pacientes; porém a sua atuação ainda é muito recente no campo hospitalar e junto com os próprios profissionais de saúde. ${ }^{11}$ A enfermagem deve estabelecer vínculo multiprofissional através da produção e divulgação de conhecimentos sobre os sentimentos dessas mulheres no pós-operatório e prestar uma assistência a essas mulheres, privilegiando a elevação em todos os segmentos de sua vida, em especial as mudanças ocorridas no aspecto psicossocial, ajudando-a na readaptação no atual estilo de vida e desempenho de papéis. $^{15}$

A maioria das mulheres desta pesquisa realizava tarefas domésticas da mesma forma que antes da cirurgia de mastectomia (90\%) e não apresentava nenhum grau de dificuldade para realizálas $(40 \%)$. Em outro estudo a atividade de manter o domicílio foi citado por $70,4 \%$ das mulheres como de desempenho moderadamente bom, com escore 7 , entre 1 a 10 (incapaz de realizar até realiza extremamente bem), coincidindo com o presente estudo. ${ }^{4}$

$$
\text { É importante ressaltar que }
$$
dependendo da extensão da mastectomia a 
mulher pode ser afetada nos movimentos que realiza com os braços, repercutindo nas tarefas do seu dia a dia e em seu papel de dona de casa. Dessa forma, realizar algumas atividades se torna difícil ou até impossível dependendo do grau de incapacidade motora causada pela mastectomia no braço acometido. ${ }^{17}$

Tais dados demonstram a importância da assistência de enfermagem em identificar as necessidades da mulher, seus sintomas, suas causas e o impacto desses no seu cotidiano, favorecendo e estimulando o seu desempenho funcional no nível absoluto, estimulando a autonomia e independência, respeitando suas limitações. ${ }^{4}$

A maioria das pesquisadas não realizou reconstrução da mama $(73,3 \%)$. Apesar de as mulheres desejarem a reconstrução, não a realizam por falta de informação; por não saberem que podem fazer a reconstrução mamária imediatamente após a mastectomia; por não quererem realizar outro procedimento cirúrgico devido ao medo, dor e sofrimento; pela cultura na qual ela está inserida; e pela indicação da cirurgia ser diferente para cada paciente. ${ }^{15}$ Igualmente, estudo português obteve que de 69 mulheres submetidas à mastectomia, $80 \%$ abstiveram-se da reconstrução mamária. ${ }^{6}$ Tal decisão, de não realizar reconstrução mamária, pode ter implicações sobre a percepção da qualidade de vida e auto estima da mulher, impactando negativamente o aspecto emocional, em comparação com aquelas que realizaram a reconstrução da mama, como demonstra estudo realizado em São Paulo, com 22 mulheres mastectomizadas. ${ }^{18}$ É sugerido como assistência de enfermagem a essas mulheres o desenvolvimento de ações que sanem suas dúvidas em relação ao procedimento de reconstrução mamária, orientando-as e proporcionando conforto e segurança em relação ao tema e à tomada de decisão.

No presente estudo, a média de escore no Inventário de Beck correspondeu a $15,3 \pm 11,04$ pontos. Pontuação inferior foi obtida em estudo que recrutou 65 mulheres após 6 meses de mastectomia (média 8,6 $\pm 6,0) .{ }^{19}$ Em outro estudo, 69 mulheres submetidas à mastectomia obtiveram média de 11,71 10,11 pontos. Embora os autores não explicitassem o tempo de realização da mastectomia, verificou-se diferença significativa quando compararam-se os escores com as mulheres do grupo sem diagnóstico de 
câncer de mama (média 3,23 \pm 2,95, $p \leq 0,001){ }^{6}$

A obtenção de escores baixos no indicativo de depressão pode ser sugerida pela média de tempo de realização de cirurgia de mastectomia, de 8,3 anos, apresentada neste estudo. Pesquisa conduzida com oito mulheres em pósoperatório (três mastectomizadas e cinco com cirurgia conservadora de mama), em Americana - SP, elucida que quanto maior o tempo de diagnóstico e tratamento, menor os níveis de sintomatologia depressiva. Tais achados podem relacionar-se com recursos de enfrentamento utilizados pelas pacientes acometidas pelo câncer de mama descritos anteriormente, como a fé, apoio familiar e conjugal, aliados à assistência da equipe de saúde e o acompanhamento psicológico recebido durante todo o processo. ${ }^{9}$

A faixa etária em que as mulheres se encontravam é outro fator a ser ponderado. Dois terços delas já eram idosas e acredita-se que a qualidade de vida e sofrimentos psicológicos melhoram significativamente com a idade para mulheres mastectomizadas, fato que está associado à mudança de valores gerada pela enfermidade, como o de aproveitar melhor os momentos e a vida. ${ }^{5}$ Tal dado demonstra que o atendimento de enfermagem deve ser realizado de forma a assistir o paciente integralmente, com ênfase nas mudanças ocorridas no campo psíquico dessas mulheres. Para isso, é necessário que o profissional domine o conhecimento técnico-científico, o que implica em uma constante busca pelo conhecimento. ${ }^{20}$

Verifica-se a necessidade do enfermeiro intervir com assistência interativa e humanizada com a equipe multidisciplinar de profissionais que cuidam e assistem as mulheres, criando dessa forma, um ambiente em que a paciente sinta-se à vontade para verbalizar seus desejos, dúvidas quanto ao enfrentamento da doença e demais sentimentos vivenciados por ela, a fim de identificar possíveis diagnósticos pósmastectomia. $^{20}$

Conclusões

$\mathrm{O}$ estudo verificou que a maior porcentagem das mulheres mastectomizadas no pós-operatório tardio possuíam média de idade de $63,4 \pm 8,9$ anos, eram viúvas, com 1 a 4 anos de escolaridade, dois filhos, grupo familiar composto por 2 e 3 pessoas e religião católica. 
As características econômicas predominantes das pacientes mastectomizadas no pós-operatório tardio eram de mulheres que não exerciam atividade remunerada, apenas antes da cirurgia de mastectomia. Dentre as que trabalhavam, executavam carga horária semanal de 20 a 40 horas. Em geral as mulheres eram aposentadas ou pensionistas e contribuíam com a família, possuíam renda bruta de um salário mínimo e moravam em casa própria.

Sobre os aspectos relacionados à saúde mental e realização de tarefas domésticas houve maior porcentagem das que não apresentavam antecedente de doença mental, não realizaram acompanhamento psicológico antes ou após a cirurgia de câncer de mama, mantinham as tarefas domésticas da mesma forma que antes da cirurgia de mastectomia, não apresentaram nenhum grau de dificuldade para realizar as mesmas e não realizaram reconstrução da mama.

A média de escore no Inventário de Beck das mulheres mastectomizadas no pós-operatório tardio correspondeu a $15,3 \pm 11,04$ pontos.

A limitação da pesquisa está associada ao fato de tratar-se de um estudo transversal, além de apresentar uma pequena amostra populacional, evidenciando a necessidade de realização de mais estudos para melhor compreender e avaliar a vida de pacientes mastectomizadas no período de pósoperatório tardio, ainda pouco abordado.

É indispensável conhecer o perfil de uma determinada população para que medidas possam ser estabelecidas, visando uma estruturação adequada, concomitantemente com um acompanhamento de ações de enfermagem que busquem assistir o ser humano em sua totalidade, com vistas a prestar uma assistência holística, individualizada e humanizada. Frente aos resultados alcançados, o estudo pode auxiliar na elucidação das características sociodemográficas e de saúde das mulheres mastectomizadas na fase tardia.

\section{REFERÊNCIAS}

1. Ministério da Saúde (Brasil). Controle do câncer de mama: documento de consenso [Internet]. Rio de Janeiro: INCA; 2004. [citado em 10 maio 2015]. Disponível em: http://bvsms.saude.gov.br/bvs/publicac oes/Consensointegra.pdf

2. Silva ACC, Anjos AT, Mascarenhas IR. Perfil epidemiológico de mulheres que realizaram mastectomia em uma 
unidade referência em Salvador Bahia. Rev Bras Saúde Funcional 2014; 1(1):31-41.

3. Gomes NS, Silva SR. Avaliação da autoestima de mulheres submetidas à cirurgia oncológica mamária. Texto \& Contexto Enferm. 2013; 22(2):509516.

4. Brito JS, Marcelino JFQ. Desempenho ocupacional de mulheres submetidas à mastectomia. Cad Ter Ocup UFSCar. 2014; 22(3):473-485.

5. Majewski JM, Lopes ADF, Davoglio T, Leite JCC. Qualidade de vida em mulheres submetidas à mastectomia comparada com aquelas que se submeteram à cirurgia conservadora: uma revisão de literatura. Ciênc Saúde Colet. 2012; 17(3):707-16.

6. Seara LS, Vieira RX, Pechorro PS. Função sexual e imagem corporal na mulher mastectomizada. Rev Int Androl. 2012; 10(3):106-12.

7. Ribeiro FE, Palma MR, Araujo MF, Egydio CA, Peixoto LN, Pelai EB et al. Perfil físico e emocional de mulheres pós-cirurgia de câncer de mama. Colloq Vitae. 2014; 6(2):102-108.

8. World Health Organization. Salud mental y desarrollo: poniendo el objetivo en las personas con problemas de salud mental como un grupo vulnerable [Internet]. Geneva: World Health Organization; 2010 [citado em 10 jun 2015]. Disponível em: http://apps.who.int/iris/bitstream/10665 /84757/1/9789962642657_spa.pdf

9. Santichi EC, Benute GRG, Juhas TR, Peraro EC, Lucia MCS. Rastreio de sintomas de ansiedade e depressão em mulheres em diferentes etapas do tratamento para o câncer de mama. Psicol Hosp. 2012; 10(1):42-67.

10. Gorestein C, Andrade L. Validation of a portuguese version of the Beck Depression Inventory and the State
Trait Anxiety Inventory in Brazilian subjets. Braz J Med Biol Res. 1996; 29(4):453-57.

11. Caporossi JAM, Ribeiro HS, MorinigoT, Campos A, Fabrizío L. Mastectomia e a incidência de transtorno de estresse pós-traumático. Psicol Saúde Doenças. 2014; 15(3):800-15.

12. Sousa ALV, Ana GS, Costa ZMB. Análise da qualidade de vida em mulheres mastectomizadas atendidas no ambulatório do HBDF. Comun Ciênc Saúde. 2014; 25(1):13-24.

13. Gasparelo C, Sales CA, Marcon SS, Salci MA. Percepções de mulheres sobre a repercussão da mastectomia radical em sua vida pessoal e conjugal. Ciênc Cuid Saúde. 2010; 9(3):535-42.

14. Haddad NC, Carvalho ACA, Novaes CO. Perfil sociodemográfico e de saúde de mulheres submetidas à cirurgia para câncer de mama. Med. HUPE-UERJ. 2015; 14(1):28-35.

15. Ibiapina ARS, Maia JM, Silva LDC, Fernandes MA, Costa Filho AAI, Fernandes RO. Aspectos psicoemocionais de mulheres pósmastectomizadas participantes de um grupo de apoio de um hospital geral. Rev Interdiscip. 2015; 8(3):135-42.

16. Fernandes AFC, Bonfim IM, Araújo IMA, Silva RM, Barbosa ICFJ, Santos MCL. Significado do cuidado familiar á mulher mastectomizada. Esc Anna Nery Rev Enferm. 2012; 16(1):27-33.

17. Silva SED, Vasconcelos EV, Santana E, Araujo SJS, Valente T, Oliveira JB et al. Câncer de mama uma doença temida: representações sociais de mulheres mastectomizadas. Rev Eletrônica Gest Saúde. 2012; 3(2):73142.

18. Furlan VLA, SabinoNeto M, Abla LEF, Oliveira CJR, Lima AC, Ruiz BFO et al. Qualidade de vida e 
autoestima de pacientes

mastectomizadas submetidas ou não a reconstrução de mama. Rev Bras Cir Plást. 2013; 28(2):264-69.

19. Giardini A, Pisoni C, Giorgi I. ICF, quality of life, and depression in breast cancer: perceived disability in diseasefree women 6 months after mastectomy. Support Care Cancer. 2013; 21(9):2453-2460.
20. Soares SGSC, Albuquerque JOL. Intervenção do enfermeiro no tratamento quimioterápico de mulheres com câncer de mama. Saúde Foco. 2014; 1(1):29-45.

RECEBIDO: 07/11/16 APROVADO: 04/09/18 PUBLICADO: 09/18 